\title{
REGA: Real-Time Emotion, Gender, Age Detection Using CNN-A Review
}

\author{
Dibya jyoti Sharma ${ }^{1}$, Sachin Dutta ${ }^{2}$, Dr. Dibya jyoti Bora ${ }^{3}$ \\ ${ }^{1,2}$ Student, School of Computing Sciences(IT), The Assam Kaziranga University, Jorhat, Assam ,India \\ ${ }^{3}$ Assistant Professor, School of Computing Sciences(IT) ,The Assam Kaziranga University, Jorhat, Assam, India \\ ${ }^{1}$ cs19mscit002@kazirangauniversity.in, ${ }^{2}$ cs19mscit004@kazirangauniversity.in, ${ }^{3}$ dibyajyotibora@kazirangauniversity.in
}

\begin{abstract}
In this paper we describe a methodology and an algorithm to estimate the real-time age, gender, and emotion of a human by analyzing of face images on a webcam. Here we discuss the CNN based architecture to design a real-time model. Emotion, gender and age detection of facial images in webcam play an important role in many applications like forensics, security control, data analysis, video observation and humancomputer interaction. In this paper we present some method \& techniques such as PCA,LBP, SVM, VIOLA-JONES, HOG which will directly or indirectly used to recognize human emotion, gender and age detection in various conditions.

Index Terms - Face detection, Emotion detection, Gender and age classifications, Convolutional Neural Network (CNN)
\end{abstract}

\section{INTRODUCTION}

$\mathrm{O}$ NE OF the most active areas in facial technology is facial features such as age, gender and emotion recognition. A lot of research has been done using deep learning methods such as ANN, CNN to determine age, gender estimation and emotion detection. Human Facial expression depicts human feelings. Human tends to change feelings, perhaps due to their mental or health conditions, in the course of lifespan. Although people are brimming with various feelings, modern psychology characterizes six basic facial expressions: happiness, sadness, surprise, fear, disgust and anger as widespread feelings. Facial muscles exercise to assist with perceiving individual's feelings. Fundamental facial consideration features are eyebrows, mouth, nose and eyes. An architecture based on the convolution Neural network $(\mathrm{CNN})$ proposed here for age, gender and emotion classification. This is one of the well known deep artificial neural networks. Convolutional Neural Network based design models are broadly utilized in classification task because of their remarkable execution in facial investigation and emotion detection. The Convolutional Neural Network includes Feature extraction which extracts Features corresponding to age, gender, emotion. Furthermore CNN includes Feature classification which classifies facial images into the correct age group, gender and emotion such as happy, sad, angry, neutral.

\section{(I) Gender and Age Classification}

Gender and age classification is the facial features that play a significant role in social interaction. Age and gender classification in face images play important task in much savvy application, for example access control, low authorization, visual observation and so on.

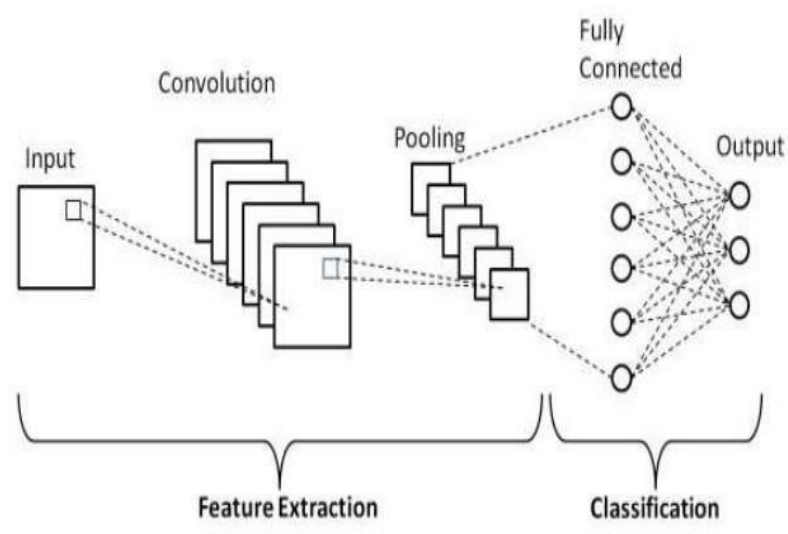

Fig. 1 Basic $\mathrm{CNN}$ architecture

\section{(II) Emotion Detection}

Emotion detection on face images can communicate and impact individual's feelings. It is intriguing and to basic comprehend the feelings passed on by the images and how the visual content of the image implies emotion.

\section{LITERATURE SURVEY}

In the past few years, several papers have successfully solved the problem of facial expression recognition So far, several tasks have been completed for real- time emotion, gender, age classification. So, Some state of artwork relevant to the proposed work is discussed in this section.

Md jashim uddin Dr. paresh Chandra Barman, khandaker Takdir Ahmed et al.[1] proposed a detection system using CNN model which can achieved $95 \%$ accuracy rate in age, gender detection with IMDB-WIKI dataset and 66\% accuracy achieved in emotion detection with FER dataset.

Thakshila R.kalansuriya and Anuuja T. Dharmaratne et al. [2] proposed a age gender detection system Using artificial neural network which achieved $70.5 \%$ accuracy rate. where FERET and FGNET datasets are used.

M.R Dileepa and Ajit Dantib et al.[3] proposed a age gender prediction system using Neural network and sigma control limit which achieved $95 \%$ accuracy rate.

Sepidehsadat et al. [4] suggested that the utilization of Gabor filter will make it simpler for the network to focus on the face, because the output direction is perfectly matched with facial wrinkles, and wrinkles will become the input of CNN. The network focuses on providing useful features with $7 \%$ age accuracy and $2 \%$ gender accuracy. 
Imane et al. [5] proposed a face detection system using HAAR cascade, CNN based on FER13(KNN) for normalization and emotion detection and Gabor binary pattern histogram sequence is used for pattern scanning. The model use for different machine learning technique (SVM, KNN, random forest and classification, regression tree) and uses KNN and SVM algorithm to obtain a high accuracy rate which reaches $70 \%$.

A better proposition is done by Rajesh et al [6] Here proposed real- time emotion detection used $\mathrm{CNN}$ with 9 layers for training and categorizing 7 different types of emotions which gives an accuracy of around 90 .

The emotion and gender detection system using CNN proposed by Md Abdullah-al-Imran et al.[7] which achieved for emotion detection $66 \%$ with FER emotion recognition dataset and with IMDB-WIKI dataset achieved 95\% for gender detection.

S L happy and Auobinda Routray et al. [8] proposed an automatic emotion recognition system using LBP classifier. Which achieved $94.39 \%$ with $\mathrm{CK}+$ dataset and the system achieved a $92.22 \%$ with JAFFE dataset .

Ramin Azarmehr, Robert Laganiere, Won-Sook Lee et al.[9] proposed a system using EDA achieved 99\% and for better accuracy and performance use support vector machine (SVM) and demographic classification strategies.

Jang-Hee Yoo, So-Hee Park and Yongjin Lee et.al[10] proposed a age gender detection system achieved 72.53\% for age detection and $98.90 \%$ for gender detection.

Octavio Arriagal and Matian Valdenegro -Toro and paul G Pl oger et al. [11] propose a Real -time emotion gender classification system using CNN which achieved $66 \%$ accuracy on emotion classification on FER-20133 dataset and with IMDB dataset achieved $95 \%$ for gender classification.

Eran Eidinger ,Roee Enbar, Tal Hassner et al. [12]

proposed a system using LBP+FPLBP which achieved accuracy $50 \%$ on age, gender Estimation of unfiltered faces.

Ajit.P Gosavi, S.R Khot et al.[13] proposed a recognition system which achieved accuracy $91.63 \%$ on JAFFE images using principal component analysis and $91.63 \%$ precision rate obtained in case of PCA.

Sidhart Nair, Dipesh Nair et al. [14] proposed a system using CNN model with HOG, adaboost algorithm and haar cascade classifier achieved $70.47 \%$ on FER- 2013 dataset for age gender and emotion classification.

Rekha N, MZ Kurian et al.[15] proposed a real time face detection system using histogram oriented gradients (HOG) and it detects the face from +90 degree to -90 degree with high accuracy rates.

Tanner Gilligan, Baris Akis et.al[16] proposed a real time emotion detection system using LeNet and AlexNet model which achieved $97 \%$ accuracy rates.

\section{Proposed Methodology}

The primary objective of the proposed methodology is to recognize the gender and age range with emotion from the human face images utilizing the set of facial features in real-

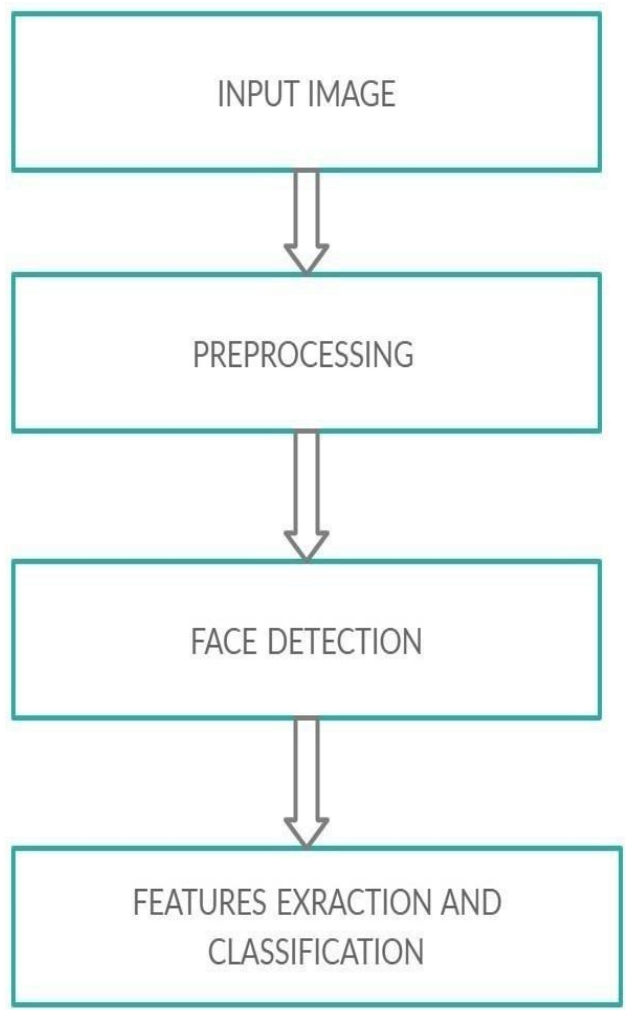

Fig-2: Flow chart of proposed methodology

time application. Feature extraction from face images is an important part of this method. In here, Fig-1 represent to show the flow of step to this methodology.

\section{(I)PRE-PROSSESSING}

The pre-processing stage can improve the quality of the input image and find the data of interest by eliminating noise and smoothing the image. It eliminates image redundancy without image details. Pre-processing also includes filtering and normalizing the image to produce a uniform size and rotated image.

\section{(II) FACE DETECTION}

The face detection process is to extract the face area from the background of Input images with various lighting conditions and complex backgrounds can be confusing and fail to recognize these expressions. It involves segmentation and extraction of facial features from the uncontrolled background.

\section{(III) FEATURE EXTRACTION}

In object detection feature extraction plays important role. Here, it includes shapes, movement, color, the texture of the facial image. It extracts meaningful information of a image compared to the original image. Feature extraction greatly reduces information of image, which has advantages in storage.

\section{(IV) FEATURE CLASSIFICATION}

The classification stage recognizes facial images and group them according to certain classes and helps them 
skilled recognition. Classification is a complicated process because it can be playing role in many areas. It classification stage also can called feature selection stage, which is deals with exchanges the retain essential information and connect them in certain parameters.

\section{POPUlar FEATURE EXTRACTION AND ClASSIFICATION METHOD}

\section{A. SVM}

It is a machine learning algorithm which is used for classification and regression. But usually, they are used for classification problems. SVM was first introduced in the 1960s ,but it was perfected in 1990 . Compared with other machine learning algorithms, SVM has its unique implementation.

The model is basically a representation of different classes in a hyper plane of a multidimensional space . SVM will generate hyper planes in an iterative manner, which can minimize errors. The goal of SVM is to divide the data set into several categories to find the maximum edge hyper plane (MMH).

\section{B. $L B P$}

The Local Binary Pattern was used for texture classification, later it was applied in other application. LBP assigns image pixel by the neighborhood (p) each pixel values within the radius $(\mathrm{R})$, represented by (gp) taking the central value $(\mathrm{gc})$ as the threshold and converting its threshold into a given decimal number label by eq. The main property of LBP is its computational simplicity, which make it analyzing possible a image in real-time.

$$
L B P_{p, R}\left(X_{C}, Y_{C}\right)=\sum_{p=0}^{p-1} s\left(g_{p}-g_{c}\right) \quad \text { where, } \mathrm{s}(\mathrm{x})=\left\{\begin{array}{lr}
1, & \mathrm{x} \geq 0 \\
0 & \text { otherwise }
\end{array}\right.
$$

\section{C. $H O G$}

Histogram of oriented gradient is feature descriptor. Feature descriptor is representations of images or Image patches, which simplify the process of extracting useful information from images and discarding redundant information. Which was proposed for object detection in various machine learning application also computer vision. HOG descriptor mainly focused on the structure of an image or object by counting the localized portion.

It is a feature extraction dense method for images. Dense means that it extracts features from all positions in the image (or regions of interest in the image), instead of only key points like SIFT in the local neighborhood.

\section{D. $P C A$}

PCA algorithm is an eigenvector method to model linear variation of high dimensional data. Principal component analysis (PCA) is the most popular appearance-based statistical method used mainly for dimensionality reduction in compression and recognition. The PCA technique is introduced by Kirby and Sirovich in 1988. This technique are used for eliminate the dimension of a face space data. The reduction of dimension helps to remove the non- essential information from input images to recognize the face. The main face images can be introduced as a feature vector or weighted sum of the Eigen's faces and stored in one - dimensional array. The PCA requires full frontal face image to be presented each time to give the good performance. The advantage of PCA is it can reduce the required data to identify individuals to $1 / 1000$ of the displayed data.

\section{E. Viola-Jones Algorithm}

The viola-Jones algorithm mainly used for object detection work that can supply a competitive rate of object detection in real-time application. Also it can be trained to detect the object classes also face detection. Three major contributions/phase of this algorithm are feature extraction, classification using boosting, Multi- scale detection algorithm . The main advantage of viola-jones algorithm, it is extremely fast feature computation $\&$ effective feature selection.

\section{CONCLUSION}

Based on this study a complete survey of the state of the art technique for age, gender and emotion classification has been reviewed and discussed via face images. Face images have become important in recent decades primarily due to their promising real-world application in several emerging fields. In this paper, various algorithms and various data set have been proposed by their researcher. Also, a summary of the published paper in this field of study was done, including the method used, their performance, and limitation. The result of this study indicates that for Real- time age, gender, and emotion HOG+viola-jones algorithm (70\%) has good accuracy rates in FER13 datasets. For only age, gender EDA (72.53\% and $98.90 \%)$ has good accuracy rates. Only for emotion using the CNN model (97\%) has the highest accuracy rates. An overall study of the contribution made on gender, emotion classification, and age estimation used to solve the real-time application problems and its application areas are forensic, security, face detection synthesis, lie detection music for mood, automated tutoring systems. In this paper most of the research work is done is in Convolutional Neural Network and algorithm like - AdaBoost, HOG, HAAR, LBP, PCA, FPLBP, and LDA which are used for age, gender and emotion recognition. By using these classifiers or algorithms we can build a system that can be claimed to be highly accurate.

\section{REFERENCES}

[1] Md. Jashim Uddin, Dr. Paresh Chandra Barman, Khandaker Takdir Ahmed S.M. Abdur Rahim, Abu Rumman Refat, Md Abdullah-AlImran6 "A Convolutional Neural Network for Real-time Face Detection and Emotion \& Gender Classification" IOSR Journal of Electronics and Communication Engineering (IOSR-JECE)

[2] Thakshila R. Kalansuriya and Anuja T. Dharmaratne,"Neural Network based Age and Gender Classification for Facial Images" International Journal on Advances in ICT for Emerging Regions

[3] M. R. Dileepa and Ajit Dantib "Human Age and Gender Prediction Based on Neural Networks and Three Sigma Control Limits" ISSN: 0883-9514 (Print) 1087-6545 (Online) Journal homepage: http://www.tandfonline.com/loi/uaai20 
[4] 2018-Sepidehsadat Hosseini, Seok Hee Lee, Hyuk Jin Kwon, Hyung Il Koo Nam Ik Cho, "Age and Gender Classification Using Wide Convolutional Neural Network and Gabor Filter", IEEE2018.

[5] Imane Lasri, Anouar Riad Solh Mourad E Belkacemi, "Facial Emotion Recognition of Students using Convolutional Neural Network", IEEE2019.

[6] Rajesh Kumar G A, Ravi Kant Kumar Goutam Sanyal, "Facial Emotion Analysis using Deep Convolutional Neural Network",2017 International Conference on Signal Processing and Communication (ICSPC). doi: $10.1109 /$ cspc. 2017.8305872 , Pg.No.- 369 to 374

[7] Md Abdullah-Al-Imran "A Convolutional Neural Network for Realtime Face Detection and Emotion \& Gender Classification" e-ISSN 2278-2834, p- ISSN: 2278-8735. Volume15, Issue 3, Ser. I (May June2020), PP 37-46.

[8] S L Happy and Aurobinda Routray“Automatic Facial Expression Recognition Using Features of Salient Facial Patches'’ DOI: 10.1109/ TAFFC. 2014. 2386334 https://rb.gy/9m5dt2.

[9] Ramin Azarmehr, Robert Laganiere, Won-Sook Lee Real-time Embedded Age and Gender Classification in Unconstrained Video https://rb.gy/pnvd2n.
[10] Jang-Hee Yoo, So-Hee Park, and Yongjin Lee "Real-Time Ageand Gender Estimation from Face Image" ISBN: 978-0-6480147-3-7.

[11] Octavio Arriaga1 and Matias Valdenegro-Toro and Paul G. Pl"oger Real-time Convolutional Neural Networks for emotion and gender classification."

[12] Eran Eidinger, Roee Enbar, Tal Hassner "Age and Gender Estimation of Unfiltered Faces",

[13] Ajit P. Gosavi, S. R. Khot "Facial Expression Recognition Using Principal Component Analysis" ISSN: 2231-2307, Volume-3, Issue-4, September 2013

[14] Sidharth Nair, Dipesh Nair, "Detection of Gender, Age and Emotion of a Human Image using Facial Features" e-ISSN: 2395-0056 www.irjet.net p-ISSN: 2395-0072

[15] Rekha N, Dr.M.Z.Kurian "Face Detection in Real Time Based on HOG" international journal of advanced Research in computer engineering \& tchnology volume 3 issue 4,april2014 ISSN:2278-1323

[16] Tanner Gilligan, Baris Akis Emotion AI, Real-Time Emotion Detection using CNN " http://web.stanford.edu/class/cs231a/ prev_projects_2016/emotion-ai-real.pdf 